\title{
Tuberculosis: Factores de riesgo asociados a mortalidad en pacientes $\leq 19$ años hospitalizados en el Instituto de Medicina Tropical
}

\section{Tuberculosis: Risk factors associated with mortality in patients $\leq 19$ years hospitalized at the Institute of Tropical Medicine}

\author{
Celia Martínez de Cuellar ${ }^{1,2}$, Dolores Lovera', Luis Gatti', Limpia Ojeda', Silvio Apodaca', \\ Claudia Zarate', Antonio Arbo ${ }^{1,2}$
}

\section{RESUMEN}

Introducción: La Organización Mundial de la Salud estima que anualmente ocurren 10 millones de casos incidentes de Tuberculosis (TB) en el mundo, de los cuales $10 \%$ ocurren en niños $<15$ años. Las dificultades en el acceso a los establecimientos de salud y el retraso en el diagnóstico y tratamiento apropiados incrementan la mortalidad en el niño. Objetivos: analizar las características clínicas, laboratoriales y los factores asociados a mortalidad en $<19$ años en pacientes hospitalizados por TB en Instituto de Medicina Tropical. Metodología: estudio observacional descriptivo, retrospectivo. Se incluyeron pacientes con diagnóstico de tuberculosis, hospitalizados, durante los años 2010 a 2018. Los datos fueron obtenidos de base de datos del Departamento de Epidemiología. Las variables incluidas fueron datos demográficos, formas de presentación, diagnostico, tratamiento y factores asociados a la mortalidad. Se realizó análisis descriptivo y analítico. Se considero error alfa $<5 \%$. Resultados: Se identificaron 98 pacientes (pts) $\leq 19$ años, con edad media de $9,3 \pm 6,3$ años, $65,3 \%$ de sexo masculino. El 34,6\% con infección por Virus de la inmunodeficiencia humana, y el 23,4\% (23/98) con desnutrición. El $42,4 \%$ procedían de poblaciones con $<100.000$ hab y el $23,4 \%$ pertenecían a pueblos originarios. El 19,4\% (19/98) fallecieron. La mortalidad se asocio a vivienda en poblaciones con $<100.000$ habitantes (33,3\% vs $8,9 \%, p=0,02$. $R R=2,3$. IC 1,1-5,0). La mortalidad fue más en la forma de tuberculosis meníngea $(46,2 \%$ vs $15,3 \%, p<0,01$. $\mathrm{RR}=3,0 ; \mathrm{IC} 1,4-6,5)$. Conclusiones: La TB predominó en el sexo masculino, más del $80 \%$ presento comorbilidades. La mortalidad fue elevada y se asocio a viviendas en aéreas con $<100.000$ habitantes y la TB meníngea.

Palabras Claves: Tuberculosis, mortalidad, VIH.

\section{ABSTRACT}

Introduction The World Health Organization estimates that 10 million new cases of Tuberculosis occur annually in the world, of which $10 \%$ occur in children $<15$ years of age. Difficulties in accessing health facilities and delaying in the diagnosis and treatment increase mortality in childhood. Objectives: to evaluate the clinical and laboratory characteristics and factors associated with mortality in patients $<19$ years of age who were hospitalized for TB at the Institute of Tropical Medicine. Materials and Methods: This was a descriptive, retrospective, observational study. We included hospitalized patients diagnosed with TB during the years 2010 to 2018. The data were obtained from the Hospital Epidemiology Department's database. The variables were demographic data, clinical presentation, diagnosis, treatment and factors associated with mortality. Descriptive and analytical analysis was performed. Alpha error was considered $<5 \%$. Results: 98 patients (pts) $\leq 19$ years old were identified, with a mean age of $9.3 \pm 6.3$ years, $65.3 \%$ were male. $34.6 \%$ had human immunodeficiency virus infection, and $23.4 \%$ (23/98) had malnutrition. $42.4 \%$ lived in areas with a population with $<100,000$ inhabitants and $23.4 \%$ belonged to native people groups. $19.4 \%$ (19/98) died. Mortality was associated with living areas with a population with $<100,000$ inhabitants ( $33.3 \%$ vs. $8.9 \%, p=0.02$. RR $=2.3$. CI $1.1-5.0)$. Mortality was higher in meningeal tuberculosis presentations $(46.2 \%$ vs. $15.3 \%, \mathrm{p}<0.01$. RR = 3.0; CI $1.4-6.5)$. Conclusions: Tuberculosis predominated in males, more than $80 \%$ presented with comorbidities. Mortality was high and was associated with living in areas with $<100,000$ inhabitants and TB meningeal form.

Keywords: Tuberculosis, mortality, HIV.

${ }^{1}$ Instituto de Medicina Tropical. Asunción, Paraguay.

${ }^{2}$ Universidad Nacional de Asunción. Facultad de Ciencias Médicas. San Lorenzo, Paraguay.

Correspondencia: Antonio Arbo; Correo: antonioarbo@hotmail.com

Conflicto de interés: Los autores declaran no poseer conflicto de interés

Recibido: 10/07/2019 Aceptado: 23/07/2019

DOI: https://doi.org/10.31698/ped.46022019002

Este es un artículo publicado en acceso abierto bajo una Licencia Creative Commons CC-BY 4.0 


\section{INTRODUCCIÓN}

En la edad pediátrica, la Tuberculosis (TB) es una de las principales causas de morbimortalidaden el mundo. La Organización Mundial de la Salud (OMS), estimó para el 2018 que 10 millones de personas enfermaron de $\mathrm{TB}$, de los cuales 1 millón eran niños. Sin embargo, ese mismo año solo 233.000 casos en niños de 0 a 14 años fueron notificados; el $80 \%$ de los cuales se reportaron en niños $>5$ años ${ }^{(1)}$.

Las estrategias de control de la tuberculosis, en general se han enfocado en el adulto debido a que los mismos son potencialmente más infecciosos debido a la elevada proporción de casos bacilíferos ${ }^{(2)}$. Sin embargo, existen varios aspectos fundamentales que deben tenerse en cuenta en la edad pediátrica. En este sentido, la tuberculosis en un niño es un evento centinela que indica transmisión reciente en una comunidad y existe una mayor probabilidad de progresión de infección a enfermedad, así como una mayor probabilidad de que se desarrollen formas graves y extrapulmonares, especialmente en niños menores de 5 años ${ }^{(2)}$. Por otro lado, se ha descrito la capacidad de transmisión de la TB por niños provocando brotes de la enfermedad, tanto en escuelas como en familias ${ }^{(2-4)}$. Otro aspecto a considerar es que los niños infectados constituyen un reservorio de la enfermedad y esta situación debe ser abordada de manera a reducir el impacto de la tuberculosis a largo plazo, en el marco de la estrategia de la OMS que tiene por objetivo poner fin a la TB en el año $2035^{(1,5)}$.

En países de baja endemia, la TB pediátrica representa menos del 5\% de todos los casos de TB, mientras que en áreas de alta incidencia puede representar hasta el $20 \%-40 \%$ de los casos ${ }^{(6)}$. Por otro lado, en áreas endémicas, la TB representa un gran porcentaje de la morbilidad y mortalidad en menores de 5 años, debido a que, en esas áreas, los niños presentan las fases más avanzadas de la enfermedad. Un estudio de autopsias en Zambia demostró que la TB fue la mayor causa de muerte por enfermedades respiratorias en niños con o sin infección por el virus de la inmunodeficiencia humana $^{(7,8)}$.

Si bien se han descrito los factores de riesgo para adquirir la infección por Mycobacterium tuberculosis en el niño, existen muy pocos estudios que analizan los riesgos asociados a la mortalidad en este grupo etario. Entre los factores de riesgo para adquirir la infección se encuentran el contacto con adultos de riesgo, pobreza extrema, residir en instituciones y contacto con personal sanitario que asiste a pacientes de riesgo. Asimismo, se describen como factores que favorecen el desarrollo de formas extrapulmonares de TB la edad menor a 4 años, especialmente menores de 2 años, adolescentes, infección por VIH y estados de inmunosupresión ${ }^{(9,10)}$. En el 2017, Paraguay reportó 2579 casos de TB nuevos más recaídas, de los cuales el 14,3\% (368/2579) fue notificado en $\leq 19$ años con una mortalidad del $1,9 \%(7 / 368)^{(1)}$. Ese mismo año, en nuestro hospital, Instituto de Medicina Tropical, se registró una mortalidad de 16,6\%(2/12) en el mismo grupo de edad. Es así que el objetivo del presente estudio es determinar las características clínicas, laboratoriales y los factores de riesgo asociados a mortalidad en el Instituto de Medicina Tropical.

\section{MATERIALES Y MÉTODOS}

Diseño y población del estudio. El estudio de carácter descriptivo, observacional, retrospectivo se realizó en el Instituto de Medicina Tropical, de Asunción, Paraguay, centro de referencia de pacientes con enfermedades infecciosas, incluyendo $\mathrm{VIH} /$ Sida. Se incluyeron todos los pacientes $\leq 19$ años hospitalizados en el periodo de tiempo comprendido entre enero 2011 y diciembre 2018, con TB diagnosticados por clínica o laboratorio. Se revisaron los registros médicos de cada paciente para recolección de datos demográficos, clínicos y de laboratorio. Fueron evaluadas las siguientes variables: sexo, edad, procedencia, forma clínica de presentación, métodos de diagnóstico, evolución. Se consideraron los siguientes factores de riesgo: a) $\leq 2$ años; b) sexo; c) comorbilidad excluyendo VIH; d) VIH/Sida, e) población indígena, y g) lugar de residencia ( $<100.000$ habitantes). Así mismo se analizó la mortalidad asociada a las formas de presentación de la TB.

Asuntos éticos: Se mantuvo en todo momento el 
anonimato y la confidencialidad de la información recolectada. Este estudio no significó riesgo adicional alguno para los pacientes evaluados, debido a que los datos se obtuvieron a partir de una revisión retrospectiva de las fichas clínicas.

Análisis estadístico: los datos recolectados fueron asentados en una hoja de cálculo de Microsoft Excel y posteriormente analizados con el programa estadístico SPSS versión 11.5 para Windows. Se empleó proporciones y porcentajes para describir variables categóricas, mientras que las cuantitativas fueron resumidas utilizando medias y desviación estándar. Para la determinación de los factores de riesgo de mortalidad se usaron pruebas chi cuadrado, RR con un intervalo de confianza del 95. El nivel de significación usado fue del 5\%, considerando que había asociación cuando el valor fue inferior a 0,05 , con un intervalo de confianza del $95 \%$.

\section{RESULTADOS}

Durante el periodo de estudio se hospitalizaron 98 pacientes $\leq 19$ años con diagnóstico de Tuberculosis en el IMT. El 41,8\% (41/98) de los pacientes provenían de distritos con poblaciones de $<100.000$ habitantes Los datos demográficos se observan en la Tabla 1.

Tabla 1. Características demográficas y clínicas de pacientes con TB en el Instituto de Medicina Tropical (IMT) periodo 2011-2018.

\begin{tabular}{lc}
\hline Datos demográficos y características clínicas & \\
\hline Edad & $9,3 \pm 6,3$ \\
\hline Media DE & $\mathbf{N}(\%)$ \\
\hline Sexo M & \\
Población indígena & $64(65,3 \%)$ \\
Población VIH (+) & $23(23,4 \%)$ \\
Patología de base & $34(34,6 \%)$ \\
Desnutrición & $8(8,2 \%)$ \\
\hline
\end{tabular}

El 62,2\% de los pacientes presentaron TB pulmonar. Entre los casos de TB-extrapulmonar la meningitis $(13,2 \%)$ fue la forma de presentación más frecuente. Las frecuencias de presentación se presentan en la Tabla 2.
Tabla 2. Formas de presentación de casos de TB en el Instituto de Medicina Tropical (IMT) periodo 20112018.

\begin{tabular}{lcc}
\hline Formas de TB & $\mathbf{N}$ & \% \\
\hline TB pulmonar & 61 & 62,2 \\
\hline TB extrapulmonar & 37 & 37,8 \\
\hline Meníngea & 13 & 13,2 \\
\hline Ganglionar & 9 & 9,2 \\
\hline Miliar & 9 & 9,2 \\
\hline Intestinal & 4 & 4,1 \\
\hline Otras & 2 & 2,0 \\
\hline
\end{tabular}

En el 53,1\% de los casos fueron diagnosticados por clínica y contacto epidemiológico; en el 46,9\% de los casos el diagnóstico fue por algún método microbiológico, como se observa en la Tabla 3.

Tabla 3. Métodos de diagnóstico de TB en $>19$ años en el Instituto de Medicina Tropical (IMT) periodo 2011-2018.

\begin{tabular}{lcc}
\hline Diagnóstico & N & $\%$ \\
\hline Criterios clínicos y epidemiológicos & 52 & 53,1 \\
\hline Métodos laboratoriales & 46 & 46,9 \\
\hline Baciloscopía & 10 & 10,2 \\
\hline Cultivo & 19 & 19,4 \\
GeneXpert & 15 & 15,3 \\
\hline Anatomía Patológica & 2 & 2,0 \\
\hline Mas de un método laboratorial & 10 & 10,2 \\
\hline
\end{tabular}

En la Tabla 4, se observa el tratamiento y evolución de los pacientes con TB.

Tabla 4. Tratamiento y evolución de los pacientes con TB en el Instituto de Medicina Tropical (IMT) periodo 2011-2018.

\begin{tabular}{lcc}
\hline Características & $\mathbf{N}$ & $\%$ \\
\hline Tratamiento HRZE* & 98 & 100 \\
Medía de hospitalización (días) \pm DS & $12,4 \pm 12,6$ & \\
Fallecidos & 19 & $19,4 \%$ \\
\hline${ }^{*}$ Isoniazida (H) Rifampicina (R) Piraziamida (Z) y Etambutol (E)
\end{tabular}

Finalmente, se analizó los factores de riesgo asociados a mortalidad por TB. Se consideraron la edad, el sexo, vivir en aéreas con población de $<100.000$ habitantes, infección por VIH y la presencia de desnutrición. Se observó un riesgo aumentado de mortalidad en aquellos pacientes procedían de comunidades con $<100.000$ habitantes $(p=0,02$. $\mathrm{RR}=2,3$. IC $95 \%=1,1-5,0)$, y en pacientes con $\mathrm{TB}$ meníngea $(p<0,01$. $R R=3,0$. IC $95 \%=1,4-6,5)$ (Tabla 5 y6). 
Tabla 5. Factores de riesgo asociados a mortalidad en pacientes con TB $\leq 19$ años en el Instituto de Medicina Tropical (IMT) periodo 2011-2018.

\begin{tabular}{lccccc}
\hline Factor de riesgo & Total & Fallecidos & & & \\
\hline$\leq 2$ años & $\mathbf{N = 9 8}$ & $\mathbf{N = 1 9}$ & $\mathbf{p}$ & RR & (IC 95\%) \\
Sexo femenino & 20 & $4(20 \%)$ & 0,06 & 0,8 & $(0,3-2,4)$ \\
Área $<100.000$ habit. & 24 & $10(41,6 \%)$ & 0,06 & 2,09 & $(0,9-4,6)$ \\
Población indígena & 42 & $14(33,3 \%)$ & 0,02 & 2,3 & $(1,1-5,0)$ \\
VIH & 23 & $6(26 \%)$ & 0,3 & 1,5 & $(0,6-3,5)$ \\
Desnutrición & 34 & $6(17,6 \%)$ & 0,7 & 0,8 & $(0,4-2,1)$ \\
\hline
\end{tabular}

Se analizo la forma de presentación en relación la localización de la infección y la asociación con la mortalidad (Tabla 6).

Tabla 6. Formas de presentación de la TB y mortalidad en pacientes con TB $\leq 19$ años en el Instituto de Medicina Tropical (IMT) periodo 20112018.

\begin{tabular}{|c|c|c|c|c|c|}
\hline Factor de riesgo & $\frac{\text { Total }}{N=98}$ & $\frac{\text { Fallecidos }}{N=19}$ & p & RR & IC \\
\hline Extrapulmonar & 37 & $10(27,0 \%)$ & 0,1 & 0,8 & $0,8-4,1$ \\
\hline Meníngea & 13 & $6(46,2 \%)$ & $<0,01$ & 3,0 & $1,4-6,5$ \\
\hline Miliar & 9 & $2(22,2 \%)$ & 0,5 & 1,5 & $0,4-5,9$ \\
\hline
\end{tabular}

\section{DISCUSIÓN}

El presente estudio reporta nuestra experiencia en la atención de pacientes $\leq 19$ años con TB, en los cuales analizamos las características clínicas, evolución y factores de riesgo asociados a mortalidad en pacientes hospitalizados en el IMT en el periodo enero 2011 a diciembre de 2018.

Nuestro estudio demostró que existe una mayor proporción de pacientes con de TB de sexo masculino, lo cual coincide con la literatura internacional ${ }^{(1,11-13)}$. Muchos pacientes tenían una condición de comorbilidad. Así, la tercera parte de los pacientes con TB tenían como condición de base una infección por VIH/Sida y la cuarta parte de los mismos presentaba desnutrición.

Una gran proporción de los pacientes presentó TB extrapulmonar. Esto es entendible dada la característica de la serie (edad pediátrica) y al tipo de población que atiende nuestro hospital, el cual es un centro de referencia de enfermedades infecciosas, y principalmente centro de concentración de pacientes con VIH/Sida. Varios estudios han demostrado una mayor proporción de casos de TB extrapulmonar en niños ${ }^{(12,14,15)}$. Así, Bisero y $\operatorname{col}^{(14)}$, reportan la presentación de las formas extrapulmonares en el $23 \%$ de los casos reportados, Gava C et al. ${ }^{(15)}$ en el 20\% de los casos de TB en niños indígenas y Vazquez Rosales y col..$^{(12)}$, lo reportan en el $69,9 \%$ de los casos reportados en un hospital de referencia.

La mortalidad por TB en los 9 años de estudio fue, superior a la mortalidad a nivel nacional $(11,5 \%)$, probablemente por ser un centro de referencia e incluir un porcentaje elevado de pacientes con coinfección TB-VIH. La mortalidad en niños con coinfección fue inferior a la reportada en el país $(30 \%-$ $40 \%$ ) en los últimos 5 años.

El presente estudio permitió identificar que la residencia en áreas con $<100.000$ habitantes tenían un riesgo 2 veces mayor a fallecer. Estudios estudios realizados en pacientes adultos con $\mathrm{TB}$, han demostrado como predictores de mortalidad la coinfección TB-VIH (OR 2,7 IC95\% 1,63-4,54), ser hombre (OR 1,4 IC95\% 1,09-1,89), la localización pulmonar (OR 1,5 IC95\% 1,13-1,95) y la edad mayor a 45 años (OR 8,9 IC95\% 5,16-15,67) ${ }^{(16)}$.

Nuestro estudio demostró además un riesgo de mortalidad incrementado asociado a la TB meníngea. Este aspecto amerita consideración. En una serie de casos de tuberculosis en pacientes pediátricos atendidos en un hospital de tercer nivel, que incluyó 93 niños, las formas clínicas más frecuentes fueron la TB pulmonar $(30.1 \%)$, ganglionar $(24.7 \%)$, miliar/diseminada (16.1\%), meníngea (13\%) y ósea $(7.5 \%)$ y no registraron fallecidos ${ }^{(12)}$. Asimismo, Bisero E y col. no registraron fallecidos en una serie de 30 niños con diagnóstico de $\mathrm{TB}^{(14)}$.

La meta de éxito de tratamiento de la OMS es de $>90 \%$, teniendo en cuenta que el manejo de la $\mathrm{TB}$, no requiere de una gran infraestructura hospitalaria, requiere de personal entrenado para la sospecha diagnóstica precoz y tratamiento oportuno. En Paraguay, se cuenta con una red de diagnóstico laboratorial, $\mathrm{RRHH}$ responsables de TB en todos los establecimientos de salud y medicamentos proveídos por el Programa Nacional de Control de TB. Sin embargo, la elevada mortalidad observada y su asociación con el área de 
residencia de los pacientes, así como con el desarrollo de formas extrapulmonares, pone de manifiesto la debilidad del sistema de salud del país, la falta de aplicación de normas de TB para el abordaje de la TB latente y la falta de entrenamiento de los pediatras para abordar esta patología.

Este estudio tiene las limitaciones del escaso número de pacientes y la no realización de un estudio de regresión múltiple, para una mejor discriminación de algunos factores de riesgo que no llegaron a ser significativos en el estudio bivariado. Sin embargo, muestra que la TB sigue constituyendo un grave problema de salud pública en el Paraguay, donde la mortalidad por la enfermedad en el niño se encuentra asociada a las debilidades del sistema de salud. Nuestro estudio sugiere la importancia de la óptima implementación de la estrategia de la OMS "Fin de la TB" lo cual requiere del fuerte compromiso de las autoridades de salud y otros sectores del país.

\section{REFERENCIAS BIBLIOGRÁFICAS}

1. World Health Organization. World Health Organization Report 2018. WHO; 2018.

2. Nelson LJ, Wells CD. Global epidemiology of childhood tuberculosis. Int J Tuberc Lung Dis. 2004; 8(5):636-647.

3. Alvarez-Castillo MC, Cano Escudero S, Taveira Jimenez JA. Microepidemias de tuberculosis en centros escolares. ¿Cómo seleccionar los contactos? Gac Sanit. 2007; 21(6):465-470.

4. Nelson JL, Moreno A, Orcau A, Alnet N, Martinez-Roig A, Cayla JA et al. Transmission of childhood tuberculosis risk factors associated with an unidentified index case and outbreak evolution in Barcelona (1987-2007). Pediatr Infect Dis J. 2010; 29(9):876-879.

5. Haas W. High time to tackle childhood tuberculosis. Euro Surveill. 2011;16(12):pii=19827.

6. Donald P, Maher D, Qazi S; Stop TB Department and the Department of Child and Adolescent Health and Development of the World Health Organization. A research agenda for childhood tuberculosis. Improving the management of childhood tuberculosis within national tuberculosis programmes: research priorities based on a literature review. WHO; 2007. Disponible en: http://apps.who.int/iris/bitstream/10665/ 69611/1/WHO HTM TB 2007.381 eng.pdf

7.- Chintu C, Mudenda V, Lucas S, Nunn A, Lishimpi K, Maswahu D, et al. Lung diseases at necropsy in African children dying from respiratory illnesses: a descriptive necropsy study. Lancet. 2002; 360:985-90.

8. Marais BJ, Schaaf HS. Childhood tuberculosis: an emerging and previously neglected problem. Infect Dis Clin North Am. 2010; 24:727-49.

9. Bermejo M, Clavera I, Michel de la Rosa F, Marín B. Epidemiología de la tuberculosis. An Sist Sanit Navar.
2007;30(Supl. 2):7-19.

10. Low S, Ang LW, Cutter J, James L, Chee CB, Wang YT, et al. Mortality among tuberculosis patients on treatment in Singapore. Int J Tuberc Lung Dis. 2009; 13(3):328-34.

11. Ramos JM, Pérez-Butragueño $M$, Tesfamariam A, Reyes F, Tiziano G, Endirays J, et al. Comparing tuberculosis in children aged under 5 versus 5 to 14 years old in a rural hospital in southern Ethiopia: an 18-year retrospective cross-sectional study. BMC Public Health. 2019; 19:856.

12.-Vázquez Rosales SG, Acosta Gallegos C, Miranda Novales MG, Fuentes Pacheco C, Labra Zamora MG, Pacheco Rosas $\mathrm{O}$, et al. Análisis de una serie de casos de tuberculosis en pacientes pediátricos atendidos en un hospital de tercer nivel. Bol. Med. Hosp. Infant. Mex. 2017; 74(1):27-33.

13. Starke R. Tuberculosis (Mycobacterium tuberculosis). In Kliegman R, Stanton B, Schor N, Behrman R, Geme J, eds. Tratado de Pediatría. 19 ed. Philadelphia: WB Saunders Elsevier; 2011.p. 1040-56.

14. Bisero E, Luque G, Borda ME, Melillo K, Zapata A, Varela S. Tuberculosis en una población pediátrica atendida en un hospital público. Adherencia al tratamiento. Estudio descriptivo. Rev. Amer. Med. Respiratoria. 2013; 13(4).

15. Gava C, Malacarne J, Giraldo Rios DP, Sant'Anna CC, Bastos Camacho LA, Basta PC. Tuberculose em crianças indígenas da Amazônia brasileira. Rev. Saúde Pública. 2013;47(1).

16. Rodríguez-Valín, E, Villarrubia Enseñat S, Díaz García O, Martínez Sánchez EV. Factores de riesgo asociados a los resultados potencialmente insatisfactorios y a la mortalidad durante el tratamiento antituberculoso en España. Rev. Esp. Salud Publica. 2015; 89(5). 